\title{
SENSITIVITY ANALYSIS OF AVALANCHE PHOTODIODE AND PIN DIODE DETECTORS IN OPTICAL RECEIVERS
}

\author{
PROMISE ELECHI ${ }^{* 1}$, SUNNY ORIKE ${ }^{1}$, WINNER MINAH-EEBA ${ }^{1}$, CHIKERENMA \\ EZINNE IKPO ${ }^{1}$
}

${ }^{l}$ Department of Electrical Engineering, Rivers State University, Port Harcourt, Nigeria

\begin{abstract}
This research explained how to establish the optical receiver sensitivity and the link power budget design in terms of quality factor, receiver power and bit error rate (BER) for several values of attenuation at $100 \mathrm{~km}$ fiber length. The design parameters which were taken into consideration were a constricted transmitter, Zender modulator, attenuator, lowpass Bessel filter, Non-Return-to-Zero modulation format, CW laser, avalanche photodiode (APD) and PIN diode detectors and the type of network coding, Bit Error Rate Analyzer (BER) etc. The objective was achieved using optisystem 7 professional simulation software layout to design the power system budget and optical receiver circuit, the avalanche photodiode and PIN diode were used as the detector. Optisystem simulations were used to determine the optimum power and the receiver sensitivity for several values of attenuation. This research investigated and analyzed the performance of optical receiver at a wavelength of $1550 \mathrm{~nm}$. Simulations were made and the Bit Error Rate analyzer values for the various parameters taken. Several values of Q-Factor and Bit Error Rate were tabulated, and the corresponding graphs plotted. There was an improvement in the quality factor, the quality of signal with a low noise value and the extended transmission or propagation time was reduced.
\end{abstract}

Keywords: APD, PIN, BER, BER Analyzer, Optisystem

\section{INTRODUCTION}

Optical fiber is a dielectric wave guide or medium in which information (voice, data or video) is transmitted through glass or plastic fiber, in the form of light [1]. It consists of the transmitter, optical lines and a receiver. The use of light wave for communication purposes could be related to ancient days if we consider optical communication in a wider view. It could be recalled that optical communication is any communication system that utilizes light for communication. A major advancement took place in 1972 when a scientist called Claude Chappe developed a system for transmitting mechanically coded messages through long distance using intermediate relay stations. His invention was called optical telegraph. Light was used to make the coded signals visible so that the relay could intercept them [2].

The visible optical waves or light has been commonly used for communication purpose for many years. Among all the indices used to analyze the performance of a group of optical receivers, a receiver sensitivity is a fundamental factor that best describe the minimum optical power needed for the optical receiver to get a sufficient SNR. A receiver is said to have a good sensitivity if it achieves the same function with less optical power incident [3].

\footnotetext{
${ }^{*}$ Corresponding author, email: elechi.promise@ @ust.edu.ng

(C) 2018 Alma Mater Publishing House
} 
Photodiodes are semiconductor devices that are responsive to high energy particles and photons. It operates by absorbing photons and produces a motion of current in an external circuit [4]. The output of an optical transmission system has a receiver device which decodes the information carried by the optical signal. Photodetector is the first component when considering a receiver. The photodetector detects the light power falling upon it and at the same time converts the light variation of the optical power into a corresponding changing electric current. The two common photodiode use are PIN and APD. Worthy of note is the fact that different materials and characteristic of the material being used can affect the system performance. Two main aspect must be considered when selecting the photodetector; the compatibility and the performance $[2,5]$.

The function of the receiver is to convert the optical signal from the fiber to electrical signal. Optical receiver consists of signal processing circuitry, an amplifier and a Photodetector [5]. The first thing a receiver does when signal is received is to convert the optical signal into an electrical signal. Thereafter, the signal is amplified to an optimum level so that the following process can be done. It is necessary to determine and predict the characteristics of the system based on mathematical models of many receiver stages in order to design a receiver.

In 2012, an optical receiver was designed using trans-impedance amplifier in MOS technology [6]. The year 2017 saw another invention that records the sensitivity in an optical preamplified receiver by joining Polarization Division Multipleing-Quadrature Phase Shift Key (PDM-QPSK) and 16-PPM (16- Pulse Position Modulation) with a pilot assisted digital coherent detection. The system obtained a 3dB sensitivity merits over PDM-QPSK in an optically preamplified receiver. This possibility was made with respect to an integrated active thin film head for high density recording (digital recording). However, it has problem arising from the joining of thin film and standard semiconductor process [7].

[8], while analyzing the characteristics of optical receiver circuit invented optical receiver circuit with active equalizer. The invention relates specifically to the optical receiver circuits with a means for regulating the input signal amplitude. The optical receiver has the signal input section, called frontend in most cases and a signal amplifying and shaping unit called linear channel. The shaping of signal is executed by an amplifier with an automatic gain control (AGC) embedded in it followed by an amplifier with an equalizer circuit. This receiver has a linear channel unit having an active equalizer regulated by the photocurrent of the input network [5] and [9].

\section{MATERIALS AND METHOD}

\subsection{Description of Method}

The design system is divided into major categories. The first part is a transmitter designed in a constricted pattern. The pseudo-Random Bit sequence generator direct the non-return-to-zero (NRZ) and the message signal and is responsible for the conversion of the message signal into unipolar signal format and a laser which generate laser signal with $1550 \mathrm{~nm}$ at $0 \mathrm{dBm}$.In the second stage the electrical signal is modulated into optical signal by a MECAH ZENDER modulator and is transmitted via the fiber line. The third stage consist of the optical signal attenuated by an attenuator at the receiving end. The system uses a cascaded optical receiver that convert the optical signal into electrical signal and have an APD and PIN detectors connected in parallel, and in series with a low pass Bessel filter $(0.75 \times$ bit rate) all in constricted form. The signal is finally detected by a Bit Error Rate Analyzer to observe the quality factor, eye diagram BER.

\subsubsection{Quality Factor}

The Quality Factor (Q-Factor) is the measure of the extent to which a noisy pulse is for diagnostic purposes. The eye diagram pattern oscilloscope will automatically generate information to indicate the Q-factor number. A higher number (Q-factor) in the result indicates that the pulse is relatively noise free.

$$
Q(d B)=20 \log \sqrt{S N R} \sqrt{\frac{B_{o}}{B_{C}}}
$$

$B_{0}$ is the optical bandwidth of the photodetector;

$B_{c}$ is the electrical bandwidth of the receiver filter. 
Thus,

$$
Q(d B)=S N R+10 \log \frac{B_{o}}{B_{C}}
$$

from equation 2, $\mathrm{Q}$ is proportional to SNR.

In Optisystem, general noise computations were done by the optical spectrum analyzers or the sampling oscilloscope. The measurements were carried over a particular measuring range.

If $B_{0}<B_{c}$, then $S N R(d B)>Q(d B)$. For practical designs $S N R(d B)>Q(d B)$ by at least 1-2 dB. When designing a high bit rate system, the receiver margin is approximately $2 \mathrm{~dB}$ in such a way that $\mathrm{Q}$ is about 2 dB smaller than SNR $(\mathrm{dB})[1]$.

\subsubsection{Eye Diagram}

An eye pattern display (Figure 1) when an oscilloscope is coupled and driven by an optical receiver output and is activated by the signal source driving the transmitter. When the eye is opened more, it signifies good optical signal quality. It is time domain based and allows for waveform distortion displayed immediately the simulation is activated. The Bit error rate Analyzer measures the performance of the system based on the signal before and after the propagation [10].

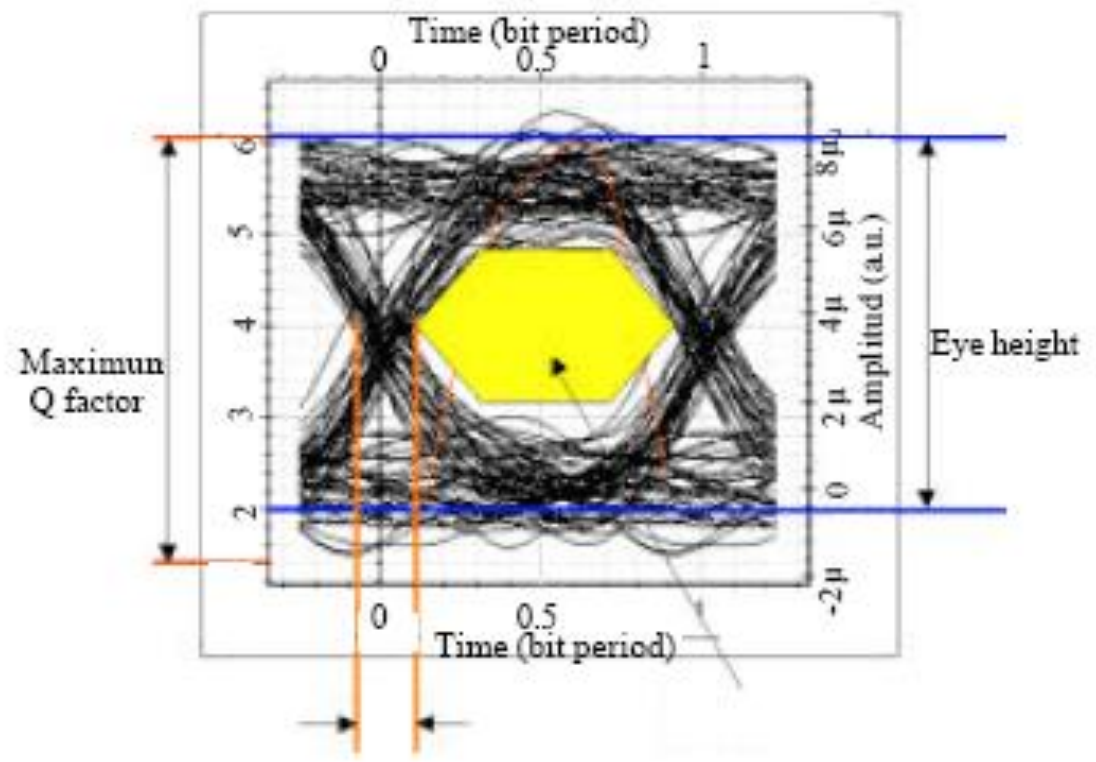

Fig. 1. Eye Diagram Parameter [10].

\subsubsection{Attenuation on Optical Link}

Attenuation is the reduction in power of the light signal as it is transmitted or the reduction of signal strength during transmission. It is the opposite of amplification, and is common when a signal is transmitted from one point to another. If the signal attenuation is high, it becomes incoherent. Attenuation is measured in decibels. Signal attenuation is defined as the ratio of the optical input/transmitted power $(\mathrm{Pi})$ to the optical output power (Po). Optical input power is the power injected into the fiber from an optical source. Optical output power is the power received at the fiber end or at the optical detector. The following equation defines the signal attenuation as unit of length.

$$
\text { Attenuation }=\left(\frac{10}{L}\right) \log _{10}\left(\frac{P i}{P o}\right)
$$




\subsubsection{System Designed Power Budget}

System designed power budget is simply the result obtained after subtracting the optical receiver sensitivity from the transmitted Power. Power budget is measured in decibels $(\mathrm{dB})$. The system designed in Figure 3 was for the system power consumption rate. Values were obtained and tabulated. For every value of attenuation, the receiver power was measured. It was observed that the attenuation and the optical receiver power were directly proportional.

From the optimization of the power budget shown in Figure 2, at $100 \mathrm{~km}$ fiber length, $1550 \mathrm{~nm}$ for the wavelength and the laser diode, the highest attenuation the system could cope with was $52.1094 \mathrm{~dB} / \mathrm{km}$ with a Q-Factor of 5.94811 (approximately 6) or 5.94808 (simulated value) and a BER of 1.25083E-9 dB under the influence of an amplifier. A Q-Factor of 6 and a BER of any number to the power of -9 (E-9) was the standard for optimum performance at $100 \mathrm{~km}$ of fiber length (Figure 4).

System Designed Power Budget $=($ Transmitted Power $)-($ Receiver Sensitivity $)$

\begin{tabular}{|c|c|c|c|}
\hline \multicolumn{2}{|l|}{ Parameter: } & \multicolumn{2}{|l|}{ Result: } \\
\hline Parameter & Value & Result & Value \\
\hline Optical Attenuator-Attenuation & 20 & BER Analyzer-Max. Q Factor & 44.7759 \\
\hline Optical Attenuator-Attenuation & 80 & BER Analyzer-Max. Q Factor & 0 \\
\hline Optical Attenuator-Attenuation & 50 & BER Analyzer-Max. Q Factor & 8.33634 \\
\hline Optical Attenuator-Attenuation & 65 & BER Analyzer-Max. Q Factor & 0 \\
\hline Optical A.ttenuator-Attenuation & 57.5 & BER A.nalyzer-Max. Q Factor & 0 \\
\hline Optical Attenuator-Attenuation & 53.75 & BER Analyzer-Max. Q Factor & 4.48804 \\
\hline Optical Attenuator-Attenuation & 51.875 & BER Analyzer-Max. Q Factor & 6.184 \\
\hline Optical Attenuator-Attenuation & 52.8125 & BER Analyzer-Max. Q Factor & 5.28209 \\
\hline Optical Attenuator-Attenuation & 52.3438 & BER Analyzer-Max. Q Factor & 5.71924 \\
\hline Optical Attenuator-Attenuation & 52.1094 & BER Analyzer-Max. Q Factor & 5.94811 \\
\hline Optical Attenuator-Attenuation & 51.9922 & BER Analyzer-Max. Q Factor & 6.06517 \\
\hline Optical A.ttenuator-Attenuation & 52.0508 & BER Analyzer-Max. Q Factor & 6.00642 \\
\hline
\end{tabular}

Fig. 2. Optimization of the System Power Budget.

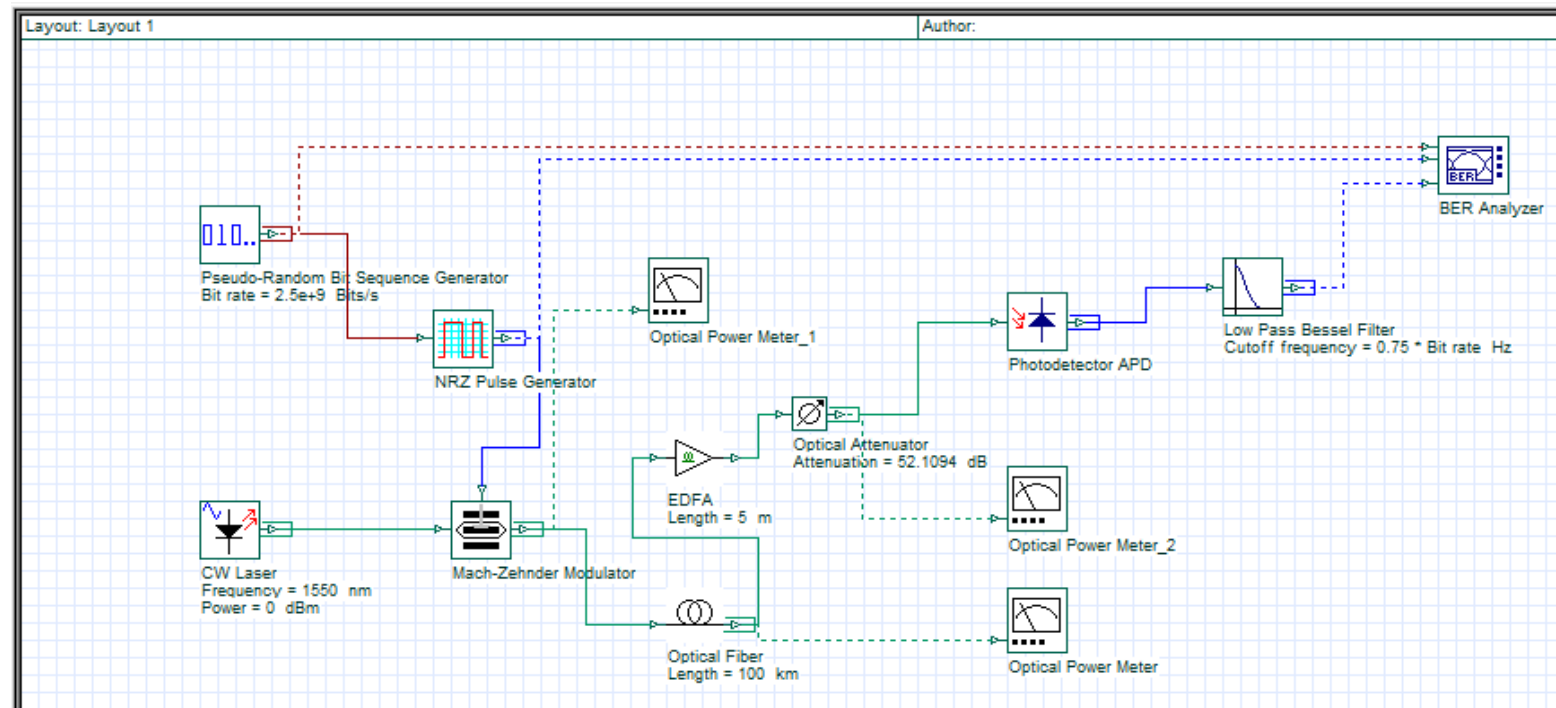

Fig. 3. System Design Power Budget. 


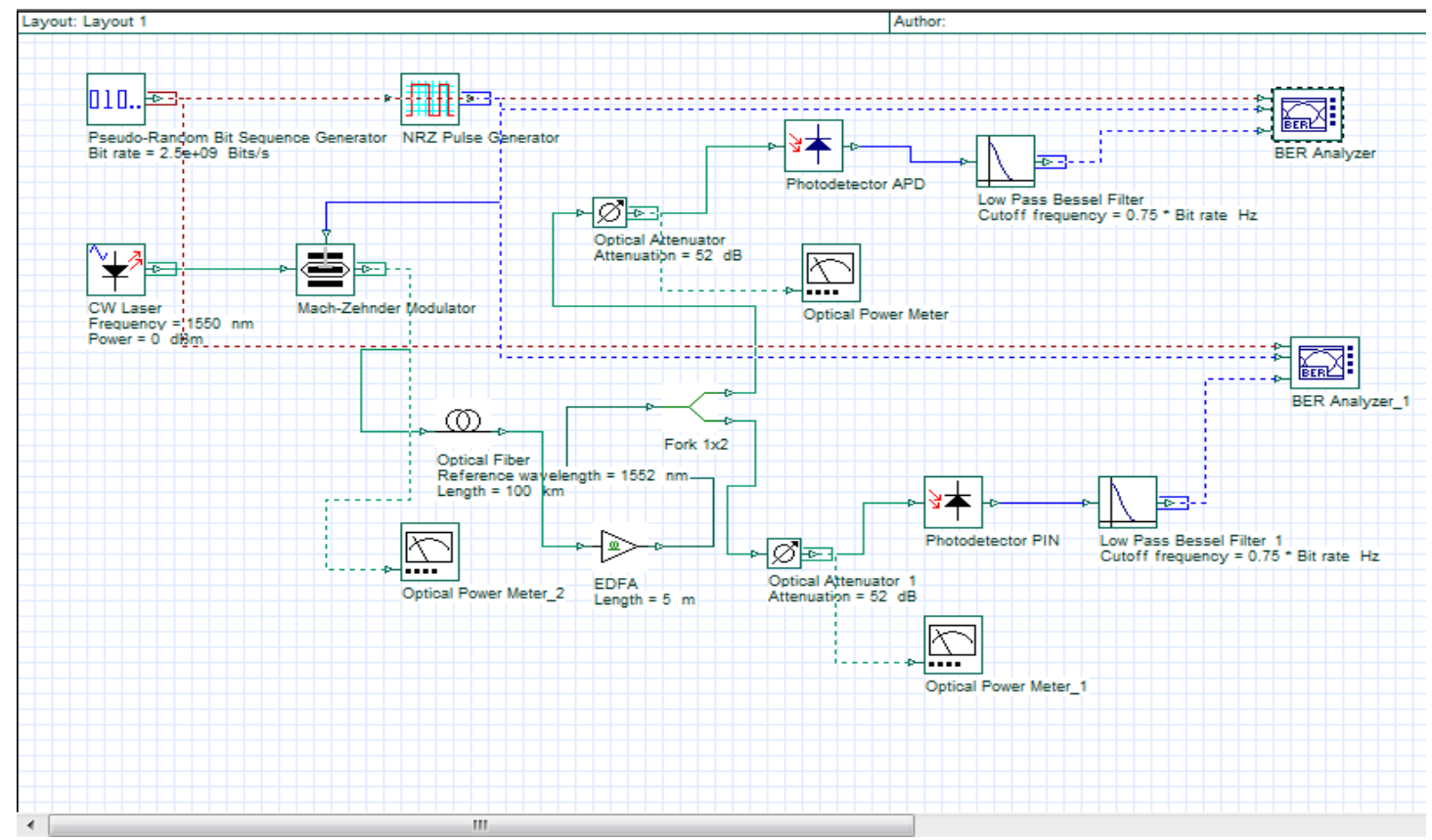

Fig. 4. System Design for the Receiver Sensitivity Input Power.

\section{RESULTS AND DISCUSSION}

The BER analyzer in Figures 8 and 9 show the value of the BER and the Q-factor for APD and PIN photodetectors when the attenuation was $30 \mathrm{~dB} / \mathrm{km}$. Figure 10 show optical power for APD and PIN at $30 \mathrm{~dB} / \mathrm{km}$ (Figure 7). The values obtained by varying the attenuation were shown in Tables 1 and 2.

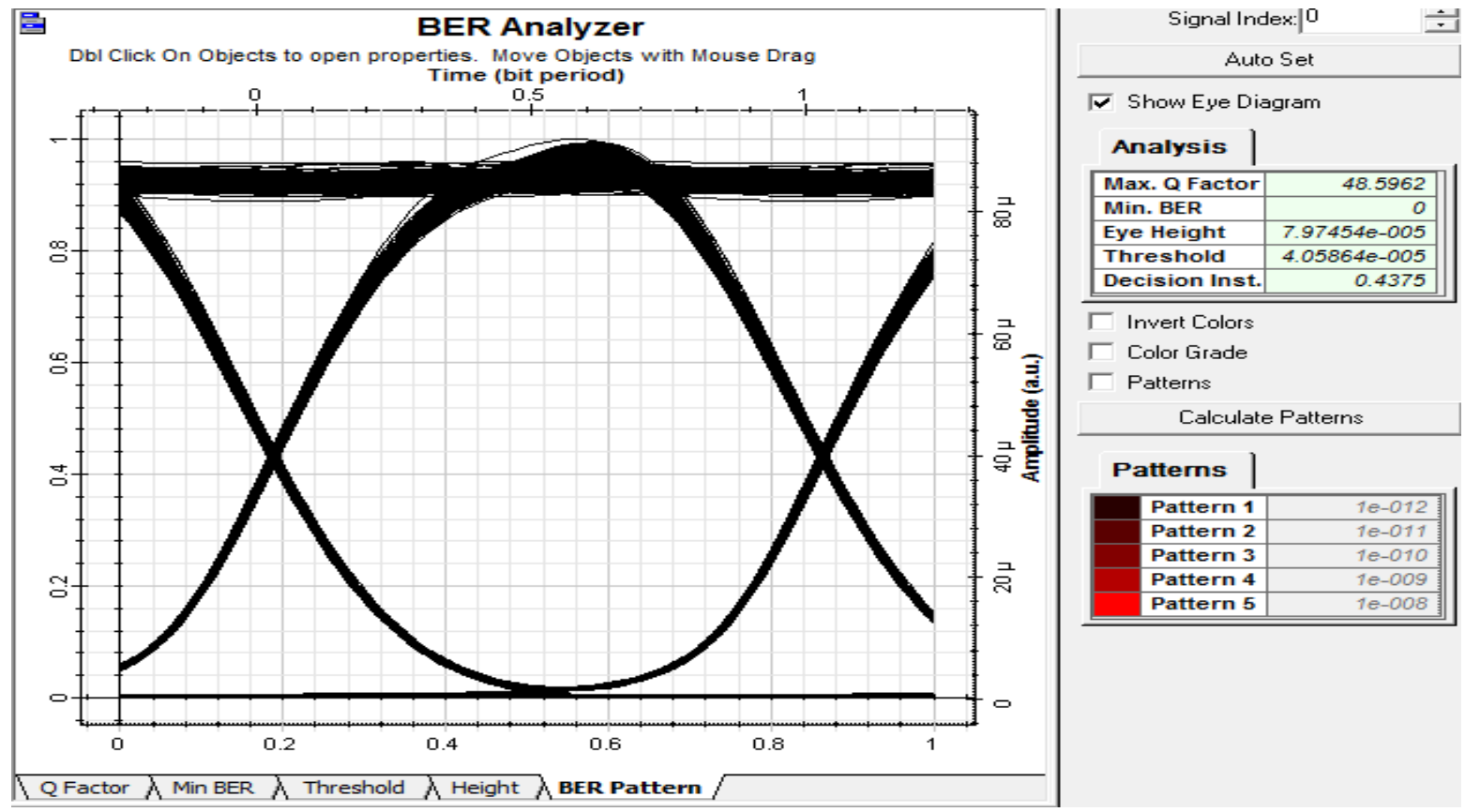

Fig. 5. Receiver Sensitivity of APD at $30 \mathrm{dBm} / \mathrm{km}$. 


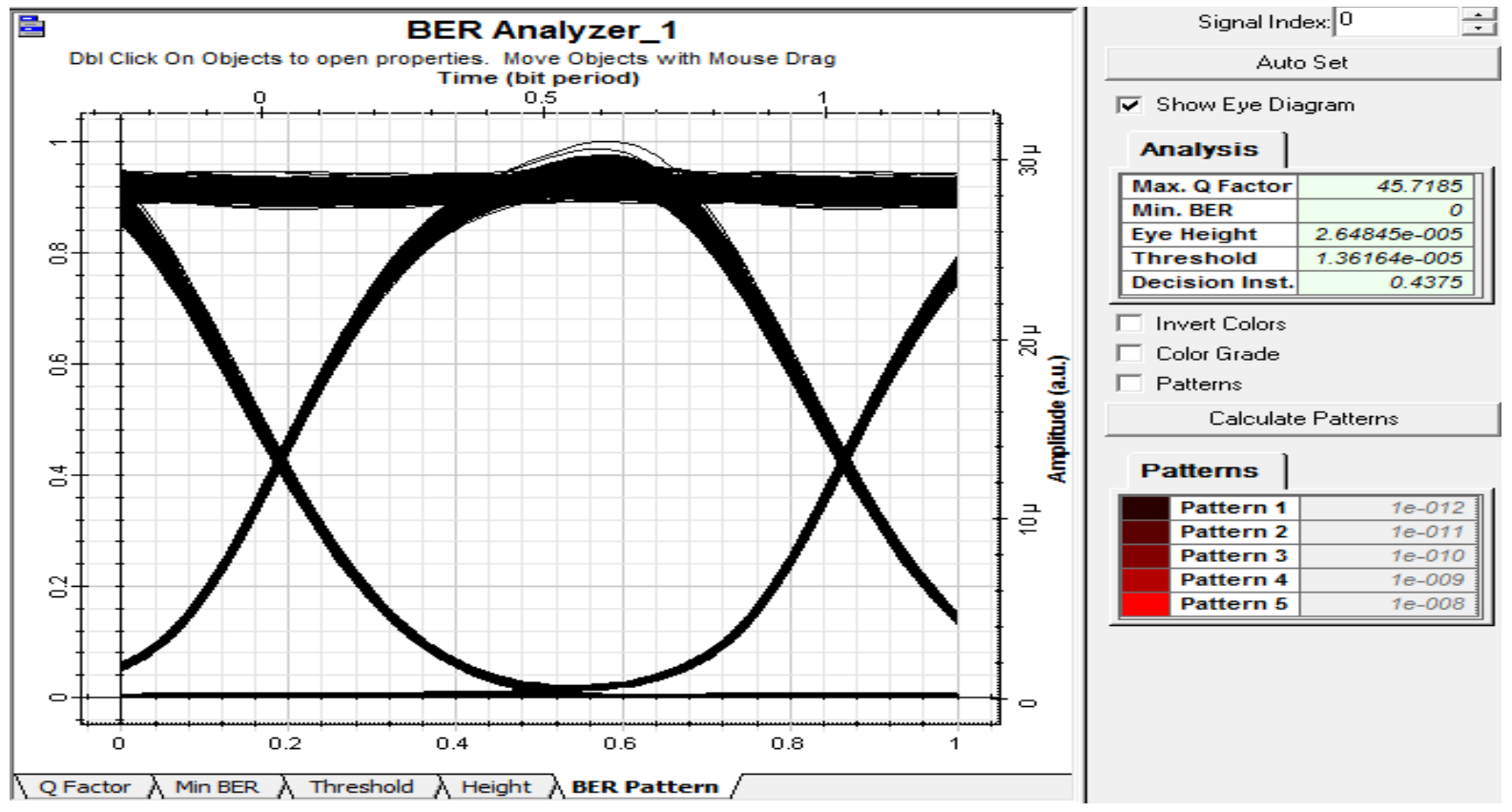

Fig. 6. Receiver Sensitivity of PIN at $30 \mathrm{dBm} / \mathrm{km}$.

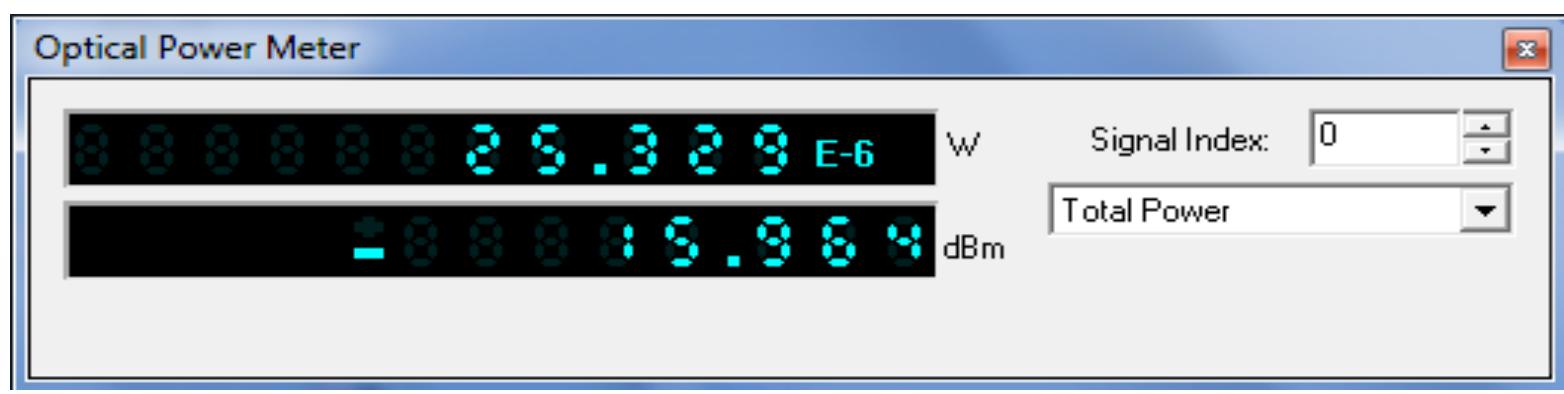

Fig. 7. Optical Power of APD and PIN at $30 \mathrm{dBm} / \mathrm{km}$.

Table 1. Variation of Power versus Q-factor and BER with PIN.

\begin{tabular}{|c|c|c|c|c|}
\hline S/N & $\begin{array}{c}\text { Attenuation } \\
(\mathrm{dB} / \mathrm{km})\end{array}$ & $\begin{array}{c}\text { Receiver Power } \\
(\mathrm{dBm})\end{array}$ & Q-Factor & BER(dB) \\
\hline 1 & 30 & -15.96 & 45.72 & 0 \\
\hline 2 & 32 & -17.96 & 44.75 & 0 \\
\hline 3 & 34 & -19.96 & 43.08 & 0 \\
\hline 4 & 36 & -21.96 & 40.24 & 0 \\
\hline 5 & 38 & -23.96 & 35.81 & $3.69 \mathrm{E}-281$ \\
\hline 6 & 40 & -25.96 & 29.80 & $1.69 \mathrm{E}-195$ \\
\hline 7 & 42 & -27.96 & 23.03 & $1.22 \mathrm{E}-117$ \\
\hline 8 & 44 & -29.96 & 16.64 & $1.73 \mathrm{E}-62$ \\
\hline 9 & 46 & -31.96 & 11.44 & $1.27 \mathrm{E}-30$ \\
\hline 10 & 48 & -33.96 & 7.62 & $1.29 \mathrm{E}-14$ \\
\hline 11 & 50 & -35.96 & 4.99 & $3.00 \mathrm{E}-7$ \\
\hline 12 & 52 & -37.96 & 3.13 & $8.7 \mathrm{E}-4$ \\
\hline 13 & 54 & -39.96 & 0 & 1 \\
\hline 14 & 56 & -41.96 & 0 & 1 \\
\hline
\end{tabular}


Table 2. Variation of Power versus Q-factor and BER with APD.

\begin{tabular}{|c|c|c|c|c|}
\hline S/N & $\begin{array}{c}\text { Attenuation } \\
(\mathrm{dB} / \mathrm{km})\end{array}$ & Receiver power $(\mathrm{dB})$ & Q-Factor & BER(dB) \\
\hline 1 & 30 & -15.96 & 48.60 & 0 \\
\hline 2 & 32 & -17.96 & 48.57 & 0 \\
\hline 3 & 34 & -19.96 & 48.48 & 0 \\
\hline 4 & 36 & -21.96 & 48.28 & 0 \\
\hline 5 & 38 & -23.96 & 47.73 & 0 \\
\hline 6 & 40 & -25.96 & 46.43 & 0 \\
\hline 7 & 42 & -27.96 & 43.59 & 0 \\
\hline 8 & 44 & -29.96 & 38.32 & $1.38 \mathrm{E}-321$ \\
\hline 9 & 46 & -31.96 & 30.64 & $2.06 \mathrm{E}-206$ \\
\hline 10 & 48 & -33.96 & 22.21 & $1.26 \mathrm{E}-106$ \\
\hline 11 & 50 & -35.96 & 15.03 & $2.49 \mathrm{E}-51$ \\
\hline 12 & 52 & -37.96 & 9.82 & $4.48 \mathrm{E}-23$ \\
\hline 13 & 54 & 39.96 & 6.33 & $1.19 \mathrm{E}-10$ \\
\hline 14 & 56 & -41.96 & 4.04 & $2.64 \mathrm{E}-5$ \\
\hline 15 & 58 & -43.96 & 0 & 1 \\
\hline
\end{tabular}

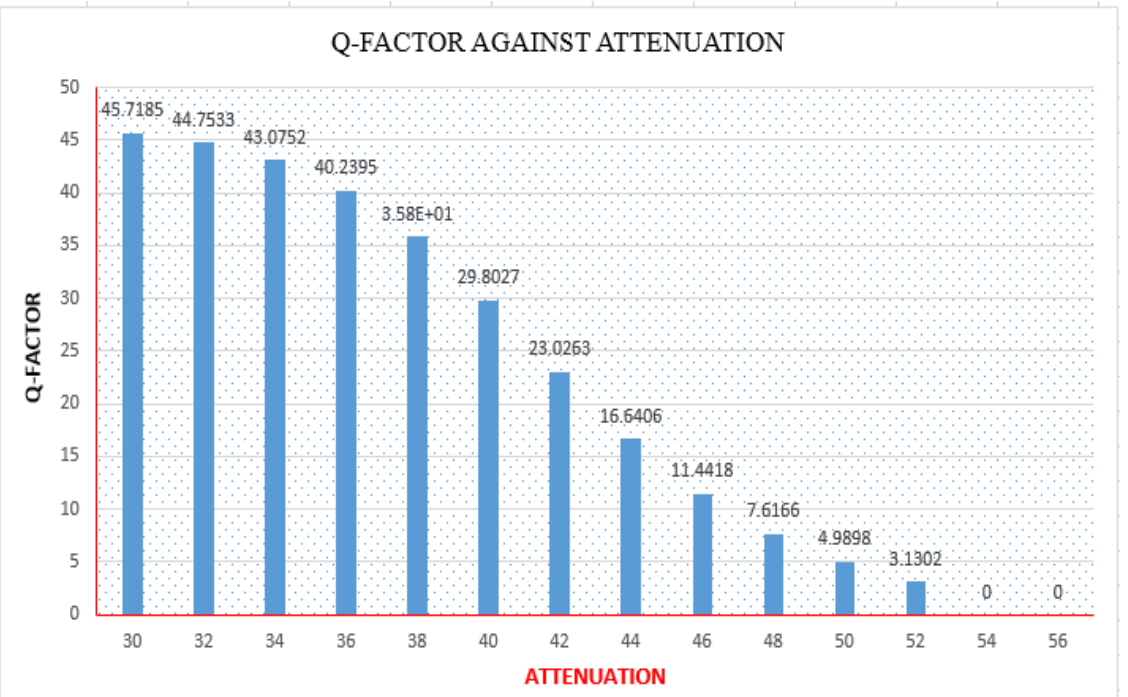

Fig. 8. Graph of Attenuation and Q-Factor for PIN Diode.

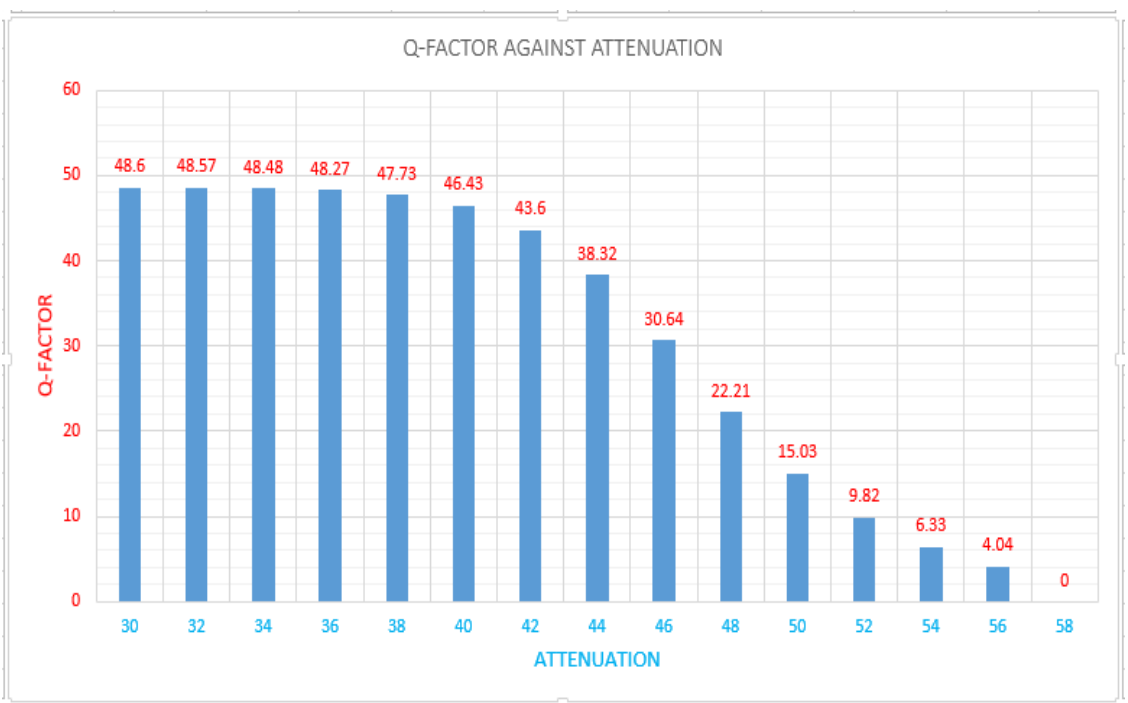

Fig. 9. Graph of Attenuation and Q-Factor for APD. 


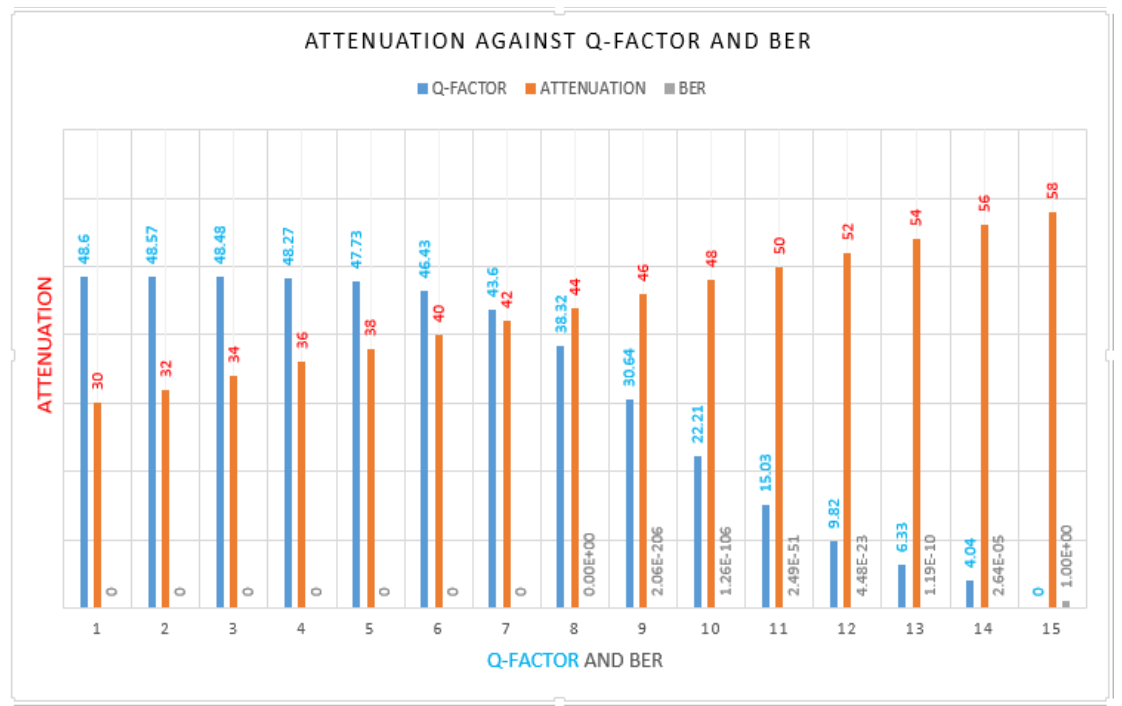

Fig. 10. Attenuation versus BER and Q-Factor for APD.

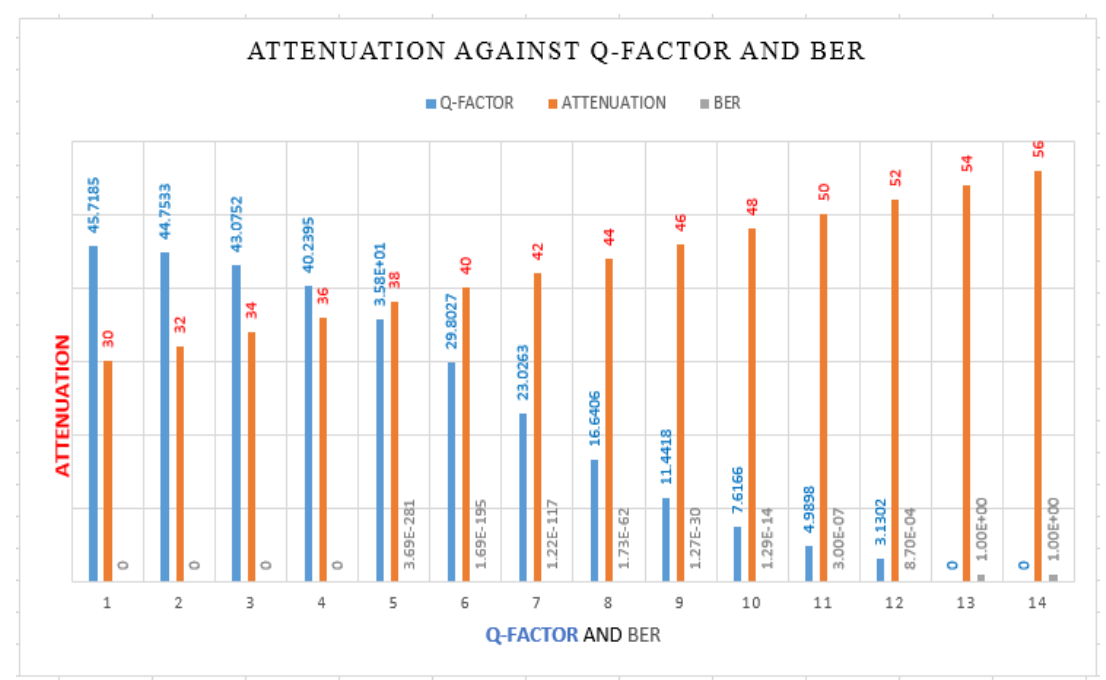

Fig. 11. Attenuation versus BER and Q-Factor for PIN.

Figures 8 and 9 show the graphical representation of the quality factor against attenuation. It was observed that attenuation and Q-Factor are inversely proportional. As the attenuation increases the quality of signal degrade. Table 1 and 2 show that APD detector has a higher Q-factor than PIN photodetector for the same value of attenuation. In addition, APD has wider eye opening that PIN (Figures 5 and 6). This result show that APD has better signal improvement that PIN photodetector. From Figures 10 and 11, it was observed that attenuation and the Bit error rate increases sequentially against the Q-Factor. For every same value of attenuation, the Q-factor of APD was higher than that of PIN diode. This characteristic of APD make it an excellent device for sensitivity improvement.

\subsection{Bit Error Rate Mathematical Formulation}

Let $P_{1}$ be the Power at level 1, $P_{0}$ be the Power at level 0. Emax $=$ Maximum Eye Amplitude, Eeye $=$ Maximum Eye Opening.

It is important to note that we consider the probability of having 1 or 0 when the output was at $50 \%$ crossing.

The optical receiver decision circuit compared the sampled value with a threshold $\left(\mathrm{P}_{\mathrm{AVG}}\right)$ and called it bit 1 if $P_{1}>\mathrm{P}_{\mathrm{AVG}}$ or bit 0 if $P_{1}<\mathrm{P}_{\mathrm{AVG}}$. Error occurs if $P_{1}>\mathrm{P}_{\mathrm{AVG}}$ for bit 0 and $P_{1}<\mathrm{P}_{\mathrm{AVG}}$ for bit 1 . 


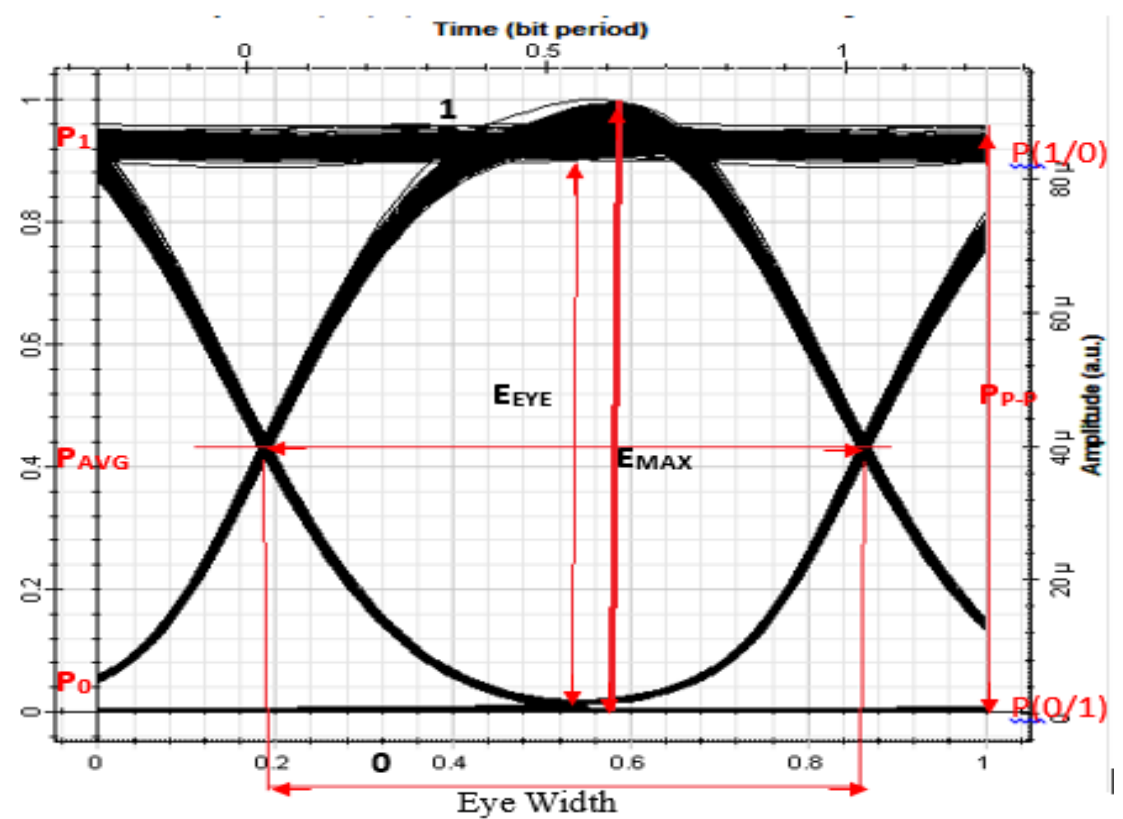

Fig.12. Modeling BER using Eye Diagram at $30 \mathrm{~dB} / \mathrm{km}$.

These errors can be included by specifying the bit error rate or the error probability as:

$$
\mathrm{BER}=\mathrm{p}(1) \mathrm{P}(0 / 1)+\mathrm{p}(0) \mathrm{P}(1 / 0)
$$

$\mathrm{p}(1)$ is the probability of receiving a $1 \mathrm{bit}, \mathrm{p}(0)$ is the probability of receiving a 0 bit;

$\mathrm{P}(0 / 1)$ is the conditional probability of deciding 0 when a 1 is sent, $\mathrm{P}(1 / 0)$ is the conditional probability of deciding 1 when a 0 is sent.

At $\mathrm{P}_{\mathrm{AVG}}$ (average power) which is $50 \%$ crossing, $\mathrm{P}(1 \mathrm{~s})$ and $\mathrm{P}(0 \mathrm{~s})$ are equal.

$$
\text { Thus } \mathrm{P}(1)=\mathrm{P}(0)=\frac{1}{2}
$$

Substitute equation (6) into (5)

$$
\mathrm{BER}=\frac{1}{2}[\mathrm{P}(0 / 1)+\mathrm{P}(1 / 0)]
$$

The Gaussian Probability Density Function (PDF) is given as:

$$
f_{X}(x)=\frac{1}{\sigma \sqrt{2 \pi}} e^{-(x-m)^{2}} / 2 \sigma^{2}
$$

Applying equation (8) to the BER analyzer in Figure 12.

$$
\mathrm{P}(0 / 1)=\frac{1}{\sigma_{1} \sqrt{2 \pi}} e^{-\left(P_{A V G}-P 1\right)^{2}} / 2 \sigma_{1}{ }^{2}
$$

Taking the integral of equation (9): 


$$
\mathrm{P}(0 / 1)=\frac{1}{\sigma_{1} \sqrt{2 \pi}} \int_{-\infty}^{P_{A V G}} e^{-\left(P_{A V G}-P 1\right)^{2}} / 2 \sigma_{1}{ }^{2} \mathrm{dP}_{\mathrm{AVG}}
$$

$$
\text { Let } \mathrm{P}_{\mathrm{AVG}}=\mathrm{Pa}
$$

Where $\mathrm{P}_{\mathrm{AVG}}=\mathrm{Pa}$ is the Average Power.

$$
\begin{gathered}
\mathrm{P}(0 / 1)=\frac{1}{\sigma_{1} \sqrt{2 \pi}} \int_{-\infty}^{P_{a}} e^{-\left(P_{a}-P 1\right)^{2}} / 2 \sigma_{1}^{2} \mathrm{dPa} \\
\text { Let } \mathrm{Z}=\frac{(P 1-P a)}{\sigma 1 \sqrt{2}} \\
\mathrm{P}(0 / 1)=\frac{1}{\sigma_{1} \sqrt{2 \pi}} \int_{\infty}^{\frac{P 1-P a}{\sigma 1 \sqrt{2}}} e^{-Z^{2}}(-\sigma 1 \sqrt{2} d Z) \\
\mathrm{P}(0 / 1)=-\int_{\infty}^{\frac{P 1-P a}{\sigma 1 \sqrt{2}}} \frac{1}{\sqrt{\pi}} e^{-Z^{2}} d Z \\
\mathrm{P}(0 / 1)=\frac{1}{\sqrt{\pi}} \int_{\frac{P 1-P a}{\sigma 1 \sqrt{2}}}^{\infty} e^{-Z^{2}} d Z \quad \mathrm{P}(0 / 1)=\frac{1}{2} x \frac{2}{\sqrt{\pi}} \int_{\frac{P 1-P a}{\sigma 1 \sqrt{2}}}^{\infty} e^{-Z^{2}} d Z
\end{gathered}
$$

From equation (16) let:

$$
\mathrm{K}=\frac{P 1-P a}{\sigma 1 \sqrt{2}}
$$

Substitute equation (17) into equation (16):

$$
\mathrm{P}(0 / 1)=\frac{1}{2} x\left(\frac{2}{\sqrt{\pi}} \int_{K}^{\infty} e^{-Z^{2}} d Z\right)
$$

But the Error Function is given as:

$$
\operatorname{erfc}(Z)=\frac{2}{\pi} \int_{Z}^{\infty} e^{-Z^{2}} d Z
$$

Put $Z=K$ and substitute into equation (19):

$$
\operatorname{erfc}(K)=\frac{2}{\pi} \int_{K}^{\infty} e^{-Z^{2}} d K
$$

Substitute equation (20) into (18):

$$
\mathrm{P}(0 / 1)=\frac{1}{2} \operatorname{erfc}(K)
$$

Substitute equation (17) into equation (21):

$$
\mathrm{P}(0 / 1)=\frac{1}{2} \operatorname{erfc}\left(\frac{P 1-P a}{\sigma 1 \sqrt{2}}\right)
$$




$$
\mathrm{P}(1 / 0)=\frac{1}{2} \operatorname{erfc}\left(\frac{P a-P o}{\sigma 0 \sqrt{2}}\right)
$$

From equation 4.3BER $=\frac{1}{2}[\mathrm{P}(0 / 1)+\mathrm{P}(1 / 0)]$.

Substituting equations (22) and (23) into equation (7):

$$
\mathrm{BER}=\frac{1}{4}\left[\operatorname{erfc}\left(\frac{P 1-P a}{\sigma 1 \sqrt{2}}\right)+\operatorname{erfc}\left(\frac{P a-P o}{\sigma 0 \sqrt{2}}\right)\right]
$$

The shot and thermal (Johnson) noise can be modeled using Gaussian Statistics. From equation (3):

$$
I_{t n}=\sqrt{\left(I_{s n}^{2}+I_{j n}^{2}\right)}
$$

If the total variance in bit level for $\mathrm{P}_{1}$ is $\sigma_{1}$ then:

$$
\begin{aligned}
& \sigma_{t n}=\sqrt{\left(i_{S, 1,0}^{2}+i_{T, 1,0}^{2}\right)} \\
& \sigma_{1,0}^{2}=\left(i_{S, 1,0}^{2}+i_{T, 1,0}^{2}\right)
\end{aligned}
$$

The total variance in bit level for $\mathrm{P}_{0}$ is $\sigma_{0}$ :

$$
\sigma_{0,1}^{2}=\left(i_{S, 0,1}^{2}+i_{T, 0,1}^{2}\right)
$$

Where $\mathrm{I}_{\mathrm{S}, 1.0}$ is the shot noise at bit level $1, \mathrm{I}_{\mathrm{S}, 0,1}$ is the shot noise at bit level $0, \mathrm{I}_{\mathrm{T}, 1,0}$ is the thermal noise at bit level 1.

$\mathrm{I}_{\mathrm{T}, 0,1}$ is the thermal noise at bit level $0, \sigma^{2}{ }_{1,0}$ is the total variance in bit level for $\mathrm{P}_{1}, \sigma^{2}{ }_{0,1}$ is the total variance in bit level for $\mathrm{P}_{0}$.

\section{CONCLUSION AND RECOMMENDATIONS}

\subsection{Conclusion}

This study is to compare the performance of APD and PIN detector in enhancing the sensitivity of optical receiver. The system power budget and optical receiver circuit were modeled and simulated using optisystem and the system performance was analyzed. The bit error rate analyzer gives a visual feedback on the quality of the optical signal as well as the numerical analysis of the quality of the signal. Optimum sampling time corresponding to the maximum eye-opening yield the best protection against noise. From the simulation we observed that the quality of the signal depends on the bit error rate. The higher the value of the bit error rate the poorer the signal quality. The simulation show that APD gave higher Q-Factor than PIN photodiode. The use of APD improved the optical quality of the signal.

\subsection{Recommendations}

In view of the simulations and conclusion of the research, the following recommendations are to be considered for continues improved sensitivity. To improve the receiver response, the range of values from serial numbers 17 in Table 1 should be used to design the optical receiver shown in Figure 12. 


\section{REFERENCES}

[1] Alamo, A., Microelectronics devices and circuits, United States, Springer, 2005, p. 23.

[2] Govind, P., Optical communication: its history and recent progress, USA, Springer, 2004, p. 23-28.

[3] Ales, P., Influence of temperature variation on optical receiver sensitivity and its Compensation, Journal of Radioengineering, vol. 16, no. 3, 2007, p. 28-33.

[4] https://www.rp-photonics. com/photodiodes.html (2.02.2018).

[5] Elechi, P., Orike, S., Minah-Eeba ,W., Simulated sensitivity improvement of optical receiver in fiber optic network, Advances in Applied Sciences, vol. 3, no. 4, 2018, p. 43-51.

[6] Sowmya, G., Subramanyan, M.V., Sofia, P.J., Design of an optical receiver using trans-impedance amplifier with light detector and equalization in mos technology, International Journal of Computer Trends and Technology, vol. 3, no. 3, 2012, p. 412-420.

[7] Xiang, L., Wood, T.H., Tkach, R.W., Chandrasekhar, S., Demonstration of record sensitivity. Journal of Lightwave Technology, vol. 5, no. 4, 2017, p. 212-221.

[8] Abdelhakim, B., Belabbes, S., Analysis of fundamental photodetection noises and evaluation of PIN and APD photodiodes performances using an optical high debit transmission chain simulated by optisystem, International Journal of Computer Applications, vol. 115, no. 18, 2015, p. 32-40.

[9] Masud, M.A., Samsuzzaman, M., Rahman, M.A., Bit Error rate performance analysis on modulation techniques of wideband code division multiple access, Journal of Telecommunication, vol.1, no. 2, 2010, p. 2229.

[10] Aashima, B., Gaurav, S., Performance analysis of optical communication system using fiber bragg grafting, SSRG International Journal of Electronics and communication Engineering, vol. 2, no. 1, 2015, p. 874-881.

[11] Azadeh, M., Fiber optics engineering, United States, Springer, 2009, p. 374. 